\title{
Sleep problems, strengths and difficulties in elementary school children
}

\author{
Jamal Driouch, Dirk Alfer, Leonie Fricke-Oerkermann, Gerd Lehmkuhl
}

Department of Child and Adolescent Psychiatry and Psychotherapy, University of Cologne, Cologne, Germany; jamaldriouch@yahoo.de

Received 19 June 2013; revised 21 July 2013; accepted 12 August 2013

Copyright (C) 2013 Jamal Driouch et al. This is an open access article distributed under the Creative Commons Attribution License, which permits unrestricted use, distribution, and reproduction in any medium, provided the original work is properly cited.

\section{ABSTRACT}

Aim: To assess behavioral and sleep disturbances in childhood, it is necessary to implement standardized brief questionnaires for children and their parents. Especially complaints of insomnia often are underdiagnosed in pediatric medicine. Methods: We developed a sleep questionnaire (KöSI-KJ) and used it together with German versions of the SDQ (parent and child reports) for epidemiologic studies in Cologne. The sleep questionnaire obtains 33 items for parents and 28 items for children. Ratings were dichotomized for this analysis in "sometimes/ often present" versus "not present". The German versions of SDQ contain 5 subscales each with 5 items. Data from a sample of 1490 children of fourth grade in elementary schools in Cologne (age ranged from 8 to 11 years) are shown. Results: Children indicate signs of sleep disturbances in higher frequency than their parents do. Concerning gender and ethnical group, there no significant differences can be shown. Children with signs of insomnia, dyssomnia, daytime sleepiness or restless sleep showed significantly higher scores for all relevant SDQ subscales in parental and childrens' reports. Children with signs of parasomnia showed higher scores concerning emotional problems and the SDQ total score. Children with enuresis showed higher scores for all SDQ subscales and the total score.

Keywords: Sleep Disorder; Behavioral Disturbance in Childhood; Correlation SDQ and Sleep Disturbance

\section{INTRODUCTION}

Children worldwide are concerned by sleep-and be- havioral disturbances [1-3]. The aetiological causes, risk factors and course of sleep and behavioral disturbances in childhood are complex [4-14]. Moreover, prevalence of psychiatric behavioral disturbance increases up to $17 \%$ and $26 \%$ in school age [15]. The need for prevention and effective treatment instrument has to be emphasized [16-18]. It has been shown that daily information and sensation sleep are indispensable [19-21], typical signs of sleeplessness are missed and diagnose might be therefore deferred in the pediatric medicine [22-24]. Sleeplessness and sleep disturbance influence child's behavior, otherwise behavioral disturbance can influence sleep customs [25-27].

The actual study presents the deficient actual condition and the need for studies and tools. Standardized brief questionnaires to comprise children's behavioral and sleep disturbance can surely facilitate diagnostical complexity for the investigator and the examine doesn't bore, analogical to clinical check-up in medical prevention.

\section{METHODS}

\subsection{Participants}

Overall, 5372 children of forth grade in schools from Cologne and their parents participated in this study. The children's age ranged from 8 to 11 years (mean 9.5 years). 1517 children were excluded because of missing data. In 1490 cases data from parents and their respective child was available for the analysis. The gender difference of the participants was equated $50.3 \%$ boys and $49.7 \%$ girls. The ethnic relation was balanced in the total sample $91 \%$ German and $9 \%$ foreign children.

\subsection{Sleep Questionnaire (KöSI-KJ)}

A German sleep questionnaire was developed (KöSIKJ-Kölner Schlafinventar für Kinder und Jugendliche). 33 items for parents and 28 for the children are related to the topics: insomnia, dyssomnia, parasomnia, restless 
legs, sleep hygiene and environment and daytime activities. Each item is answered on a three-point scale ranging from "not present" to "sometimes present" to "often present". For this analysis ratings were dichotomized in "sometimes/often present" versus "not present". The participants were asked to answer according to the last three months.

\subsection{Strengths and Difficulties Questionnaire (SDQ)}

The SDQ (Strengths and Difficulties Questionnaire) is a questionnaire developed in England by Goodman 1997 to evaluate behavioural problems and strengths of children. It was translated by a German children psychiatrist and a professional translator, and improved and standardized by Woerner W. et al. 2002. It contains a balanced degree of symptoms and positively formulated behavioural attributes. The questionnaire contains 5 subscales (prosocial behaviour, hyperactivity and attention problems, emotional problems and problems with peers), each with 5 items. Each item is answered on a threepoint scale ranging from "not true" over "somewhat true" to "certainly true".

\subsection{Study Design}

The questionnaires were dispatched to the participants in october 2005. In november 2005 all members of the survey received a reminder postcard. The questionnaire contained the sleep questionnaire, Strengths and Difficulties Questionnaire (SDQ), and also general questions about gender, birthday, age and demand consulting.

A total of 5372 children and their parents received questionnaires. Of those 1517 responded, and 1490 could be analyzed. The others were incompletely filled.

The collected data was analyzed using the statistical software SPSS. For analysis the rating scales were dichotomized in "sometimes/often present" versus "not present". Gender and ethnical group differences were computed by $C h i^{2}$-Test $\left(x^{2}-\right.$ Test $)$ by Pearson and correlation by spearman. To compare the arithmetic mean of SDQ we used t-test for independent random examination.

\section{RESULTS}

\subsection{Parent's Reports}

In this survey children with signs of disturbed sleep showed higher values concerning emotional problems, hyperactivity-inattention and the SDQ total score (Table 1). All children with signs of sleep disturbance are significantly more affected by conduct problems and peer problems. Concerning pro-social behavior parents reports show some small but significant differences as sociated with signs of insomnia, dyssomnia, daytime sleepiness or restless sleep.

Children with often occurring nightmares and pavor nocturnus show significant higher scores for emotional problems, hyperactivity-inattention and conduct problems (Table 2). Children with episodes of pavor nocturnus are significantly more affected by peer problems. Children with episodes of somnambulism show significantly higher scores for emotional problems than children without high somnambulis. They are not significantly more affected by having hyperactivity-inattention, conduct problems, peer problems and prosocial behavior. Children suffering from Enuresis Noct. show significantly higher occurence of hyperactivity-inattention and conduct problems, significantly more occurence of emotional and peer problems (Table 2). Children with bruxism are significantly more affected from hyperactivityinattention, conduct problems and peer problems.

Snoring Children show significantly more signs of hyperactivity-inattention, conduct problems and peer problems (Table 3). Children with parental reports of sleep apnea are high significant more affected by hyperactivity-inattention, conduct problems and peer problems. There is no difference to other children concerning emotional problems or prosocial behavior.

Children showing signs of restless legs syndrome are high significant more affected by emotional problems, hyperactivity-inattention, conduct problems and peer problems. Concerning prosocial behavior there is no difference. Children with signs of snoring, enuresis or restless legs syndrom show high significant elevated SDQ total scores.

\subsection{Children's Reports}

Childrens with signs of insomnia and restless sleep show high significantly elevated scores for all SDQ scales (emotional problems, hyperactivity-inattention, conduct problems, peer problems and prosocial behavior, Table 4).

Children with signs of dyssomnia and daytime sleepiness show high significantly elevated scores for SDQ subscales emotional problems, hyperactivity, conduct problems, peer problems and prosocial problems. Signs of dyssomnia and daytime sleepiness are associated with higher SDQ total scores, too.

Concerning parasomnia children with nightmares report high significant elevated scores for emotional problems, hyperactivity, conduct problems and peer problems (Table 5). Children suffering from Enuresis Noct. report high significant elevated scores for hyperactivity, conduct problems and peer problems and significantly elevated scores for emotional problems and prosocial behavior. Enuresis or nightmares show high significant elevated SDQ total scores. 
Table 1. SDQ and insomnia/dyssomnia (parent's reports).

\begin{tabular}{|c|c|c|c|c|c|c|c|c|c|c|}
\hline & & \multicolumn{3}{|c|}{ Total score } & \multicolumn{3}{|c|}{ Emotional Problems } & \multicolumn{3}{|c|}{ Hyperaktivity } \\
\hline & & M & $\mathrm{SD}$ & $\mathrm{p}$ & M & SD & $\mathrm{p}$ & M & SD & $\mathrm{p}$ \\
\hline \multirow{2}{*}{ Insomnia } & Yes & 8.96 & 6.04 & \multirow{2}{*}{0.000} & 2.24 & 2.06 & \multirow{2}{*}{0.000} & 3.32 & 2.7 & \multirow[t]{2}{*}{0.000} \\
\hline & No & 6.02 & 4.73 & & 1.29 & 1.61 & & 2.39 & 2.21 & \\
\hline \multirow[b]{2}{*}{ Dyssomnia } & Yes & 10.1 & 6.28 & \multirow[b]{2}{*}{0.000} & 2.52 & 2.21 & \multirow[b]{2}{*}{0.000} & 3.82 & 2.67 & \multirow[b]{2}{*}{0.000} \\
\hline & No & 6.44 & 5.02 & & 1.44 & 1.7 & & 2.51 & 2.32 & \\
\hline \multirow{2}{*}{$\begin{array}{l}\text { Daytime } \\
\text { sleepiness }\end{array}$} & Yes & 10.53 & 6.44 & \multirow{2}{*}{0.000} & 2.8 & 2.22 & \multirow{2}{*}{0.000} & 3.76 & 2.71 & \multirow{2}{*}{0.000} \\
\hline & No & 6.3 & 4.87 & & 1.37 & 1.64 & & 2.49 & 2.3 & \\
\hline \multirow{2}{*}{$\begin{array}{c}\text { Awakening at } \\
\text { night }\end{array}$} & Yes & 8.08 & 5.96 & \multirow{2}{*}{0.000} & 1.99 & 2.04 & \multirow{2}{*}{0.000} & 3.1 & 2.56 & \multirow{2}{*}{0.000} \\
\hline & No & 6.36 & 4.93 & & 1.39 & 1.66 & & 2.47 & 2.31 & \\
\hline \multirow{4}{*}{ Restless sleep } & Yes & 9.72 & 6.26 & \multirow{2}{*}{0.000} & 2.27 & 2.08 & \multirow{2}{*}{0.000} & 3.76 & 2.62 & \multirow{2}{*}{0.000} \\
\hline & No & 5.96 & 4.61 & & 1.35 & 1.65 & & 2.30 & 2.21 & \\
\hline & & \multicolumn{3}{|c|}{ Conduct problems } & \multicolumn{3}{|c|}{ Peer problems } & \multicolumn{3}{|c|}{ Prosocial behavior } \\
\hline & & $\mathrm{m}$ & $\mathrm{s}$ & $\mathrm{p}$ & $\mathrm{m}$ & $\mathrm{s}$ & $\mathrm{p}$ & $\mathrm{m}$ & $\mathrm{s}$ & $\mathrm{p}$ \\
\hline \multirow{2}{*}{ Insomnia } & Yes & 2.01 & 1.7 & \multirow{2}{*}{0.000} & 1.39 & 1.7 & \multirow{2}{*}{0.000} & 8.09 & 1.66 & \multirow[t]{2}{*}{0.000} \\
\hline & No & 1.42 & 1.39 & & 0.92 & 1.38 & & 8.48 & 1.62 & \\
\hline \multirow{2}{*}{ Dyssomnia } & Yes & 2.24 & 1.77 & \multirow{2}{*}{0.000} & 1.52 & 1.8 & 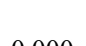 & 7.98 & 1.74 & \\
\hline & No & 1.5 & 1.45 & & 0.99 & 1.44 & & 8.41 & 1.62 & \\
\hline Daytime & Yes & 2.22 & 1.74 & 0000 & 1.74 & 1.83 & 0000 & 8.15 & 1.61 & 0048 \\
\hline sleepiness & No & 1.49 & 1.45 & 0.000 & 0.95 & 1.41 & & 8.39 & 1.64 & \\
\hline Awakening at & Yes & 1.79 & 1.6 & 0.001 & 1.21 & 1.63 & 0.010 & 8.25 & 1.64 & 0.082 \\
\hline night & No & 1.51 & 1.47 & & 0.99 & 1.44 & & 8.41 & 1.64 & \\
\hline & Yes & 2.14 & 1.73 & & 1.55 & 1.91 & & 8.09 & 0.09 & \\
\hline 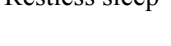 & No & 1.41 & 1.39 & 0.000 & 0.9 & 1.29 & 0.000 & 8.45 & 0.05 & 0.000 \\
\hline
\end{tabular}

Children with signs of restless legs syndrome show high significantly elevated scores for all SDQ subscales: emotional problems, hyperactivity, conduct problems, prosocial problems, total score and peer problems (Table 6).

\section{DISCUSSION}

\subsection{Association of Disturbed Sleep and Behavioral Problems}

The findings support the assumption that there are relevant associations between sleep and behavioral problems. Similarly data from other cohorts of our study showed that children with signs of insomnia, dyssomnia and daytime sleepiness are at higher risk for emotional problems, peer problems, hyperactivity and conduct problems with 2 - 3-fold higher relative risk values [28].

In a different survey, Stein et al. found high correlation between sleep disturbance in childhood and internal (anxiety, depression) and external (delinquent, aggressive) behavioral disturbance [29]. Recent surveys report about correlations between sleep disturbance and behavioral disturbance. There are no reports about the causal direction of correlation between sleep and behavioral disturbance. Behavioral disturbance is supposed to get worsened by sleep disturbance and vice versa. [10] reported about children suffering habitual snoring, who are highly significantly more affected with hyperactivity and inattention than children without habitual snoring. One year later, children who were not suffering from habitual snoring anymore showed regressive behavioral disorder (hyperactivity and inattention). Children who persisted in suffering habitual snoring were still affected with behavioral disorder. Therefore treatment of sleep disorder can reduce behavioral disorder.

Other studies report about close correlation between sleep duration and behavioral disturbance. Sleep duration 
Table 2. SDQ and parasomnia (parent's reports).

\begin{tabular}{|c|c|c|c|c|c|c|c|c|c|c|}
\hline & & \multicolumn{3}{|c|}{ Total score } & \multicolumn{3}{|c|}{ Emotional Problems } & \multicolumn{3}{|c|}{ Hyperaktivity } \\
\hline & & M & $\mathrm{SD}$ & $\mathrm{p}$ & M & $\mathrm{SD}$ & $\mathrm{p}$ & $\mathrm{M}$ & SD & $\mathrm{p}$ \\
\hline \multirow{2}{*}{ Nightmares } & Yes & 8.49 & 6.04 & \multirow{2}{*}{0.000} & 2.18 & 2.04 & \multirow{2}{*}{0.000} & 3.16 & 2.61 & \multirow{2}{*}{0.000} \\
\hline & No & 6.32 & 4.92 & & 1.66 & 0.05 & & 2.5 & 2.3 & \\
\hline \multirow{2}{*}{ Pavor nocturnus } & Yes & 11.26 & 6.05 & \multirow{2}{*}{0.000} & 2.71 & 2.24 & \multirow{2}{*}{0.000} & 3.85 & 2.45 & \multirow{2}{*}{0.000} \\
\hline & No & 6.68 & 5.19 & & 1.52 & 1.76 & & 2.61 & 2.39 & \\
\hline \multirow{2}{*}{ Somnam-bulism } & Yes & 7.96 & 5.95 & \multirow{2}{*}{0.030} & 2.09 & 2.03 & \multirow{2}{*}{0.002} & 2.85 & 2.45 & \multirow{2}{*}{0.393} \\
\hline & No & 6.81 & 5.26 & & 1.53 & 1.78 & & 2.66 & 2.4 & \\
\hline \multirow{2}{*}{ Enuresis noct. } & Yes & 9.17 & 5.98 & \multirow{2}{*}{0.000} & 2.03 & 2.2 & \multirow{2}{*}{0.037} & 3.61 & 2.8 & \multirow{2}{*}{0.001} \\
\hline & No & 6.76 & 5.26 & & 1.55 & 1.78 & & 2.62 & 2.37 & \\
\hline \multirow{4}{*}{ Bruxism } & Yes & 8.01 & 6.1 & \multirow{2}{*}{0.000} & 2.07 & 2.13 & \multirow{2}{*}{0.000} & 2.98 & 2.54 & \multirow{2}{*}{0.006} \\
\hline & No & 6.55 & 5.01 & & 1.42 & 1.66 & & 2.57 & 2.35 & \\
\hline & & \multicolumn{3}{|c|}{ Conduct problems } & \multicolumn{3}{|c|}{ Peer problems } & \multicolumn{3}{|c|}{ Prosocial behavior } \\
\hline & & M & $\mathrm{SD}$ & $\mathrm{p}$ & M & $\mathrm{SD}$ & $\mathrm{p}$ & M & $\mathrm{SD}$ & $\mathrm{p}$ \\
\hline \multirow{2}{*}{ Nightmares } & Yes & 1.88 & 1.64 & \multirow{2}{*}{0.000} & 1.27 & 1.68 & \multirow{2}{*}{0.003} & 8.3 & 1.56 & \\
\hline & No & 1.49 & 1.46 & & 0.99 & 1.42 & & 8.38 & 1.67 & 0.387 \\
\hline \multirow{2}{*}{ Pavor nocturnus } & Yes & 2.75 & 1.87 & \multirow{2}{*}{0.000} & 1.94 & 2.13 & \multirow{2}{*}{0.000} & 7.98 & 1.68 & 0032 \\
\hline & No & 1.53 & 1.47 & & 1.01 & 1.44 & & 8.38 & 1.64 & 0.032 \\
\hline Sompon bulism & Yes & 1.79 & 1.59 & 0.121 & 1.23 & 1.83 & 0240 & 8.42 & 1.67 & 0625 \\
\hline Sominam-bunism & No & 1.58 & 1.51 & 0.131 & 1.05 & 1.46 & 0.249 & 8.35 & 1.64 & 0.035 \\
\hline Fnurecis & Yes & 2.11 & 1.7 & 0001 & 1.42 & 1.73 & 0032 & 8.24 & 1.62 & 0473 \\
\hline 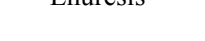 & No & 1.56 & 1.5 & 0.001 & 1.04 & 1.48 & 0.052 & 8.36 & 1.64 & $0.7 / 3$ \\
\hline Rruxism & Yes & 1.75 & 1.62 & 0035 & 1.21 & 1.67 & 0.047 & 8.24 & 1.63 & 0009 \\
\hline Dituxisint & No & 1.55 & 1.48 & 0.035 & 1.02 & 1.44 & 0.041 & 8.4 & 1.64 & 0.099 \\
\hline
\end{tabular}

Table 3. SDQ and sleep disordered breathing (parent's reports).

\begin{tabular}{|c|c|c|c|c|c|c|c|}
\hline & & \multicolumn{4}{|c|}{ Sleep disordered breathing } & \multirow{2}{*}{\multicolumn{2}{|c|}{$\begin{array}{c}\text { Restless legs Syndrom } \\
\text { Moving leg }\end{array}$}} \\
\hline & & \multicolumn{2}{|c|}{ Habitual snoring } & \multicolumn{2}{|c|}{ Sleep apnea } & & \\
\hline & & yes & no & yes & no & yes & no \\
\hline \multirow[t]{3}{*}{ Total score } & $\mathrm{M}$ & 8.18 & 6.44 & 10.74 & 6.85 & 11.29 & 6.77 \\
\hline & SD & 5.82 & 5.07 & 7.35 & 5.28 & 7.92 & 5.16 \\
\hline & $\mathrm{p}$ & \multicolumn{2}{|c|}{0.000} & \multicolumn{2}{|c|}{0.000} & \multicolumn{2}{|c|}{0.000} \\
\hline \multirow[t]{3}{*}{ Emotional Problems } & $\mathrm{M}$ & 1.86 & 1.48 & 2.59 & 1.56 & 2.94 & 1.54 \\
\hline & SD & 1.97 & 1.73 & 2.41 & 1.79 & 2.6 & 1.76 \\
\hline & $\mathrm{p}$ & \multicolumn{2}{|c|}{0.001} & \multicolumn{2}{|c|}{0.036} & \multicolumn{2}{|c|}{0.000} \\
\hline \multirow[t]{3}{*}{ Hyperactivity } & $\mathrm{M}$ & 3.15 & 2.5 & 3.96 & 2.66 & 4.27 & 2.62 \\
\hline & SD & 2.48 & 2.36 & 3.13 & 2.39 & 2.9 & 2.37 \\
\hline & $\mathrm{p}$ & \multicolumn{2}{|c|}{0.000} & \multicolumn{2}{|c|}{0.005} & \multicolumn{2}{|c|}{0.000} \\
\hline \multirow[t]{3}{*}{ Conduct problems } & $\mathrm{M}$ & 1.92 & 1.47 & 2.37 & 1.58 & 2.29 & 1.57 \\
\hline & SD & 1.61 & 1.47 & 1.92 & 1.51 & 1.9 & 1.5 \\
\hline & $\mathrm{p}$ & \multicolumn{2}{|c|}{0.000} & \multicolumn{2}{|c|}{0.008} & \multicolumn{2}{|c|}{0.001} \\
\hline \multirow[t]{3}{*}{ Peer problems } & $\mathrm{M}$ & 1.26 & 0.99 & 1.81 & 1.05 & 1.8 & 1.04 \\
\hline & SD & 1.69 & 1.42 & 2.02 & 1.49 & 2.07 & 1.47 \\
\hline & $\mathrm{p}$ & \multicolumn{2}{|c|}{0.004} & \multicolumn{2}{|c|}{0.009} & \multicolumn{2}{|c|}{0.001} \\
\hline \multirow[t]{3}{*}{ Prosocial behavior } & $\mathrm{M}$ & 8.31 & 8.37 & 8.15 & 8.36 & 7.9 & 8.37 \\
\hline & SD & 1.63 & 1.64 & 1.88 & 1.64 & 2.06 & 1.62 \\
\hline & $\mathrm{p}$ & \multicolumn{2}{|c|}{0.516} & \multicolumn{2}{|c|}{0.567} & \multicolumn{2}{|c|}{0.118} \\
\hline
\end{tabular}


Table 4. SDQ and insomnia/dyssomnia (children's reports).

\begin{tabular}{|c|c|c|c|c|c|c|c|c|c|c|}
\hline & & \multicolumn{3}{|c|}{ Total score } & \multicolumn{3}{|c|}{ Emotional Problems } & \multicolumn{3}{|c|}{ Hyperactivity } \\
\hline & & M & $\mathrm{SD}$ & $\mathrm{p}$ & M & $\mathrm{SD}$ & $\mathrm{p}$ & M & SD & $\mathrm{p}$ \\
\hline \multirow{2}{*}{ Insomnia } & Yes & 9.28 & 5.24 & \multirow{2}{*}{0.000} & 2.18 & 1.9 & \multirow{2}{*}{0.000} & 3.55 & 2.26 & \multirow[t]{2}{*}{0.000} \\
\hline & No & 6.41 & 4.38 & & 1.27 & 1.5 & & 2.52 & 2.18 & \\
\hline \multirow{2}{*}{ Dyssomnia } & Yes & 10.24 & 5.47 & \multirow{2}{*}{0.000} & 2.47 & 1.97 & \multirow{2}{*}{0.000} & 3.85 & 2.35 & \multirow{2}{*}{0.000} \\
\hline & No & 7.05 & 4.59 & & 1.48 & 1.62 & & 2.77 & 2.18 & \\
\hline \multirow{2}{*}{ Daytime sleepiness } & Yes & 11.46 & 5.39 & \multirow{2}{*}{0.000} & 2.78 & 2.01 & \multirow{2}{*}{0.000} & 4.15 & 2.31 & \multirow{2}{*}{0.000} \\
\hline & No & 6.93 & 4.47 & & 1.46 & 1.59 & & 2.76 & 2.17 & \\
\hline \multirow{4}{*}{ Restless sleep } & Yes & 9.95 & 5.44 & \multirow{2}{*}{0.000} & 2.31 & 2.01 & \multirow{2}{*}{0.000} & 3.74 & 2.37 & \multirow{2}{*}{0.000} \\
\hline & No & 7.25 & 4.73 & & 1.57 & 1.65 & & 2.84 & 2.2 & \\
\hline & & \multicolumn{3}{|c|}{ Conduct problems } & \multicolumn{3}{|c|}{ Peer problems } & \multicolumn{3}{|c|}{ Prosocial behavior } \\
\hline & & M & $\mathrm{SD}$ & $\mathrm{p}$ & M & $\mathrm{SD}$ & $\mathrm{p}$ & M & SD & $\mathrm{p}$ \\
\hline \multirow{2}{*}{ Insomnia } & Yes & 1.8 & 1.49 & \multirow{2}{*}{0.000} & 1.75 & 1.6 & \multirow{2}{*}{0.000} & 8.29 & 1.6 & \multirow[t]{2}{*}{0.002} \\
\hline & No & 1.38 & 1.24 & & 1.25 & 1.39 & & 8.56 & 1.65 & \\
\hline \multirow{2}{*}{ Dyssomnia } & Yes & 2.0 & 1.55 & \multirow{2}{*}{0.000} & 1.92 & 1.63 & \multirow{2}{*}{0.000} & 8.11 & 1.67 & \multirow{2}{*}{0.000} \\
\hline & No & 1.4 & 1.3 & & 1.36 & 1.46 & & 8.54 & 1.6 & \\
\hline \multirow{2}{*}{ Daytime sleepiness } & Yes & 2.29 & 1.61 & \multirow{2}{*}{0.000} & 2.24 & 1.74 & \multirow{2}{*}{0.000} & 7.86 & 1.87 & \multirow{2}{*}{0.000} \\
\hline & No & 1.41 & 1.26 & & 1.3 & 1.39 & & 8.58 & 1.51 & \\
\hline & Yes & 2.03 & 1.57 & & 1.87 & 1.68 & & 8.23 & 1.72 & \\
\hline Kestiess sieep & No & 1.46 & 1.3 & 0.000 & 1.39 & 1.45 & 0.000 & 8.48 & 1.59 & 0.001 \\
\hline
\end{tabular}

Table 5. SDQ and parasomnia (children's reports).

\begin{tabular}{|c|c|c|c|c|c|c|c|c|c|c|}
\hline & & \multicolumn{3}{|c|}{ Total score } & \multicolumn{3}{|c|}{ Emotional problems } & \multicolumn{3}{|c|}{ Hyperactivity } \\
\hline & & M & SD & $\mathrm{p}$ & M & SD & $\mathrm{p}$ & M & SD & $\mathrm{p}$ \\
\hline \multirow{2}{*}{ Night-mares } & Yes & 9.4 & 5.35 & \multirow{2}{*}{0.000} & 2.38 & 1.92 & \multirow{2}{*}{0.000} & 3.42 & 2.34 & \multirow{2}{*}{0.000} \\
\hline & No & 6.74 & 4.47 & & 1.23 & 1.46 & & 2.79 & 2.19 & \\
\hline \multirow{4}{*}{ Enuresis } & Yes & 10.46 & 5.21 & \multirow[b]{2}{*}{0.000} & 2.17 & 1.81 & \multirow[b]{2}{*}{0.042} & 3.95 & 2.29 & \multirow[b]{2}{*}{0.001} \\
\hline & No & 7.85 & 5.03 & & 1.75 & 1.78 & & 3.03 & 2.28 & \\
\hline & & \multicolumn{3}{|c|}{ Conduct problems } & \multicolumn{3}{|c|}{ Peer problems } & \multicolumn{3}{|c|}{ Prosocial behavior } \\
\hline & & M & SD & $\mathrm{p}$ & M & SD & $\mathrm{p}$ & M & $\mathrm{SD}$ & $\mathrm{p}$ \\
\hline \multirow{2}{*}{ Night-mares } & Yes & 1.85 & 1.44 & \multirow{2}{*}{0.000} & 1.74 & 1.61 & \multirow{2}{*}{0.000} & 8.34 & 5.35 & \multirow{2}{*}{0.10} \\
\hline & No & 1.4 & 1.32 & & 1.33 & 1.43 & & 8.48 & 4.47 & \\
\hline \multirow{2}{*}{ Enuresis } & Yes & 2.21 & 1.59 & \multirow{2}{*}{0.001} & 2.11 & 1.83 & \multirow{2}{*}{0.000} & 7.96 & 1.85 & \multirow{2}{*}{0.026} \\
\hline & No & 1.58 & 1.38 & & 1.49 & 1.51 & & 8.44 & 1.61 & \\
\hline
\end{tabular}

less than 7 hours per night is highly significantly correlated with anxiety and depression. Children who reported sleep duration of 9 hours are $30 \%$ less affected from anxiety and depression. Similar data were published in the USA and Finland concerning correlation between sleep duration and inattention/delinquent and aggressive behavioral disorder $[13,14,30]$.

Higher odds ratios are reported for children suffering 
Table 6. SDQ and restless legs (children's reports).

\begin{tabular}{|c|c|c|c|c|c|c|c|c|c|c|}
\hline & & \multicolumn{3}{|c|}{ Total score } & \multicolumn{3}{|c|}{ Emotional problems } & \multicolumn{3}{|c|}{ Hyperaktivity } \\
\hline & & M & $\mathrm{SD}$ & $\mathrm{p}$ & M & SD & $\mathrm{p}$ & M & $\mathrm{SD}$ & $\mathrm{p}$ \\
\hline \multirow{2}{*}{ RLS signs } & Yes & 10.65 & 5.2 & \multirow{2}{*}{0.000} & 2.46 & 2.02 & \multirow{2}{*}{0.000} & 3.92 & 2.24 & \multirow{2}{*}{0.000} \\
\hline & No & 7.67 & 4.97 & & 1.69 & 1.74 & & 2.98 & 2.27 & \\
\hline & & \multicolumn{3}{|c|}{ Conduct problems } & \multicolumn{3}{|c|}{ Peer problems } & \multicolumn{3}{|c|}{ Prosocial behavior } \\
\hline & & M & $\mathrm{SD}$ & $\mathrm{p}$ & M & $\mathrm{SD}$ & $\mathrm{p}$ & M & $\mathrm{SD}$ & $\mathrm{p}$ \\
\hline \multirow{2}{*}{$\begin{array}{l}\text { RLS } \\
\text { signs }\end{array}$} & Yes & 2.21 & 1.57 & \multirow{2}{*}{0.000} & 2.07 & 1.46 & \multirow{2}{*}{0.000} & 8.1 & 1.86 & \multirow{2}{*}{0.01} \\
\hline & No & 1.54 & 1.36 & & 1.71 & 1.5 & & 8.45 & 1.6 & \\
\hline
\end{tabular}

from sleep disturbance and anxiety/depression and aggressive behavioral disturbance [31,32]. Other surveys found no significant correlation between sleep and behavioral problems in childhood. Children suffering from sleep problems were more affected from internal and external behavioral disorder [25]. Significantly higher correlations to ADHS were found in children suffering from insomnia and dyssomnia with an elevated relative risk factor of 2.2. There is no significantly elevated risk factor for ADHS in children who suffer from daytime sleepiness [33]. [26] also reported that adults suffering from ADHS are significantly more affected by insomnia, dyssomnia and daily tiredness than control groups not suffering ADHS. In our survey, we found highly significant associations for all SDQ subscales (behavioral problems) for children with signs insomnia, dyssomnia, daytime sleepiness and restless sleep. Similar data were found by [34]. Children suffering from emotional problems are significantly more often affected by insomnia (34\% vs. $18 \%$ ), daytime sleepiness $(18.9 \%$ vs. $6.9 \%)$, restless sleep (32.1\% vs. $16.1 \%)$ and pavor nocturnus $(23.1 \%$ vs. $8.2 \%)$ than children not suffering emotional problems. Children suffering from hyperactivity are significantly more often affected by disrupted sleep $(39.9 \%$ vs. $15.5 \%)$, insomnia $(27.5 \%$ vs. $14.6 \%)$, bruxism $(17.7 \%$ vs. $8.9 \%)$ and somnambulism ( $25.5 \%$ vs. $10.8 \%)$. Children suffering from conduct problems significantly are more often reported with having disrupted sleep $(31.2 \%$ vs. $16.0 \%)$. Children with peer problems show significantly more daytime sleepiness (16.3\% vs. $7.2 \%)$ [35].

\subsection{Association between Parasomnia and Behavioral Disorder}

Children with episodes of pavor nocturnus, somnambulism or nightmares seem to have a two to four fold higher risk of having emotional problems, hyperactivity, conduct disorder, peer problems and an elevated SDQ total score. There is no significant association between emotional problems and peer problems. In contrast to the last mentioned survey, we did not find significant associations of pavor nocturnus and emotional disorder in our survey.

Children with enuresis are highly significantly more affected to have externalized and internalized behavioral disorders [29]. Several surveys agree on a significant association between sleep-disordered breathing and behavioral disturbance in childhood [10,36-39].

Habitual snoring conducting behavioral disorder probably is caused by arousing stimulus and dysfunction of gas exchange (children develop hypoxia and hyperkapnia) [40].

There are reports about double-rate occurrence of hyperactivity in children suffering habitual snoring [41]. In our survey we found similar results. High scores of RDI's (Respiratory Disturbance Index) in childhood measured by polysomnography are significantly associated with ADHS and aggressive and oppositional behavioral disorders. There is no significant correlation to internalized behavioral problems [39]. Children with habitual snoring and restless legs syndrome have high risk to be affected by ADHS. Still some surveys showed no significantly high risk was found for reported apnea [33]. A survey examining children suffering from ADHS by polysomnography and comparing to a healthy control group found that children suffering ADHS and sleep disturbance show similar sleep architecture as to children who only suffer sleep disturbance. Children who only suffer from ADHS but not from sleep disturbance show aberrances in sleep architecture compared to healthy children $[5,42]$. In our survey children reporting of apnea were associated with more peer problems, conduct problems and emotional problems. Children suffering RLS also show a significant correlation to have emotional problems, hyperactivity, conduct problems, peer problems and total score. [43] also report about highly significant correlation between RLS and emotional problems and about significant correlation between bruxism and emotional problems in childhood.

\section{REFERENCES}

[1] Meltzer, L.J., Johnson, C., Crosette, J., Ramos, M. and 
Mindell, J.A. (2010) Prevalence of diagnosed sleep disorders in pediatric primary care practices. Pediatrics, 125, e1410-e1418. doi:10.1542/peds.2009-2725

[2] Sadeh, A., Mindell, J. and Rivera, L. (2011) My child has a sleep problem: A cross-cultural comparison of parental definitions. Sleep Medicine, 12, 478-482. doi:10.1016/j.sleep.2010.10.008

[3] Mindell, J.A., Sadeh, A., Wiegand, B., How, T.H. and Goh, D.Y. (2010) Cross-cultural differences in infant and toddler sleep. Sleep Medicine, 11, 274-280. doi:10.1016/j.sleep.2009.04.012

[4] Gregory, A.M. and Sadeh, A. (2012) Sleep, emotional and behavioral difficulties in children and adolescents. Sleep Medicine Reviews, 16, 129-136. doi:10.1016/j.smrv.2011.03.007

[5] Wiater, A.H., Mitschke, A.R., Widdern, S., Fricke, L., Breuer, U. and Lehmkuhl, G. (2005) Sleep disorders and behavioural problems among 8- to 11-year old children. Somnologie, 9, 210-214.

[6] Owens, J.A. (2011) Update in pediatric sleep medicine. Current Opinion in Pulmonary Medicine, 17, 425-430.

[7] Keller, P. and El-Sheikh, M. (2011) Children's emotional security and sleep: Longitudinal relations and directions of effects. Journal of Child Psychology and Psychiatry, 52, 64-71. doi:10.1111/j.1469-7610.2010.02263.x

[8] Roberts, R.E., Roberts, C.R. and Chan, W. (2008) Persistence and change in symptoms of insomnia among adolescents. Sleep, 31, 177-184.

[9] Stein, M.A., Mendelsohn, J., Obermeyer, W.H., Amromin, J. and Benca, R. (2001) Sleep and behavior problems in school-aged children. Pediatrics, 107, E60. doi:10.1542/peds.107.4.e60

[10] Urschitz, M.S., Guenther, A., Eggebrecht, E., Wolff, J., Urschitz-Duprat, P.M., Schlaud, M. and Poets, C.F. (2003) Snoring, intermittent hypoxia and academic performance in primary school children. American Journal of Respiratory and Critical Care Medicine, 168, 464-468. doi:10.1164/rccm.200212-13970C

[11] Lehmkuhl, G., Fricke-Oerkermann, L., Wiater, A. and Mitschke, A. (2008) Sleep disorders in children beginning school: Their causes and effects. Deutsches Ärzteblatt International, 105, 809-814.

[12] Liu, X., Liu, L. and Wang, R.Z. (2003) Bed sharing, sleep habits, and sleep problems among Chinese school-aged children. Sleep, 26, 89-94.

[13] Paavonen, E.J., Porkka-Heiskanen, T. and Lahikainen, A.R. (2009) Sleep quality, duration and behavioral symptoms among 5 - 6-year-old children. European Child \& Adolescent Psychiatry, 18, 747-754. doi:10.1007/s00787-009-0033-8

[14] Lavigne, J.V., Arend, R., Rosenbaum, D., Smith, A., Weissbluth, M., Binns, H.J. and Christoffel, K.K. (1999) Sleep and behavior problems among preschoolers. Journal of Developmental \& Behavioral Pediatrics, 20, 164169. doi:10.1097/00004703-199906000-00005

[15] Verhulst, F.C., van der Ende, J. and Rietbergen, A. (1997) Ten-year time trends of psychopathology in Dutch children and adolescents: No evidence for strong trends. Acta
Psychiatrica Scandinavica, 96, 7-13. doi:10.1111/j.1600-0447.1997.tb09898.x

[16] Szklo-Coxe, M., Young, T., Peppard, P.E., Finn, L.A. and Benca, R.M. (2010) Prospective associations of insomnia markers and symptoms with depression. American Journal of Epidemiology, 171, 709-720. doi:10.1093/aje/kwp454

[17] Roane, B.M. and Taylor, D.J. (2008) Adolescent insomnia as a risk factor for early adult depression and substance abuse. Sleep, 31, 1351-1356.

[18] Döpfner, M., Lehmkuhl, G., Berner, W., Flechtner, H., Schwitzgebel, P., von Aster, M. and Steinhausen, H.C. (1993) Documentation of psychopathologic findings: A procedure for the evaluation of psychological disorders in children and adolescents. Z Kinder Jugendpsychiatr, 21, 90-100.

[19] Bub, K.L., Buckhalt, J.A. and El-Sheikh, M. (2011) Children's sleep and cognitive performance: A crossdomain analysis of change over time. Developmental Psychology, 47, 1504-1514. doi:10.1037/a0025535

[20] Soffer-Dudek, N., Sadeh, A., Dahl, R.E. and RosenblatStein, S. (2011) Poor sleep quality predicts deficient emotion information processing over time in early adolescence. Sleep, 34, 1499-1508.

[21] Markowitsch, H.J. (2002) Functional retrograde amnesiaamnestic block syndrome. Cortex, 38, 651-654. doi:10.1016/S0010-9452(08)70030-3

[22] Ohayon, M.M. (2008) From wakefulness to excessive sleepiness: What we know and still need to know. Sleep Medicine Reviews, 12, 129-141. doi:10.1016/j.smrv.2008.01.001

[23] Mindell, J.A. (1999) Empirically supported treatments in pediatric psychology: Bedtime refusal and night wakings in young children. Journal of Pediatric Psychology, 24, 465-481. doi:10.1093/jpepsy/24.6.465

[24] Stores, G. (1996) Practitioner review: Assessment and treatment of sleep disorders in children and adolescents. Journal of Child Psychology and Psychiatry, 37, 907925. doi:10.1111/j.1469-7610.1996.tb01489.x

[25] Goodlin-Jones, B., Tang, K., Liu, J. and Anders, T.F. (2009) Sleep problems, sleepiness and daytime behavior in preschool-age children. Journal of Child Psychology and Psychiatry, 50, 1532-1540. doi:10.1111/j.1469-7610.2009.02110.x

[26] Surman, C.B., Adamson, J.J., Petty, C., Biederman, J., Kenealy, D.C., Levine, M., Mick, E. and Faraone, S.V. (2009) Association between attention-deficit/hyperactivity disorder and sleep impairment in adulthood: Evidence from a large controlled study. Journal of Clinical Psychiatry, 70, 1523-1529. doi:10.4088/JCP.08m04514

[27] Johnson, E.O., Roth, T., Schultz, L. and Breslau, N. (2006) Epidemiology of DSM-IV insomnia in adolescence: Lifetime prevalence, chronicity, and an emergent gender difference. Pediatrics, 117, e247. doi:10.1542/peds.2004-2629

[28] Kraenz, S., Fricke, L., Wiater, A., Mitschke, A., Breuer, U. and Lehmkuhl, G. (2004) Häufigkeit und belastungsfaktoren bei schlafstörungen im einschulalter. Prax 
Kinderpsychol Kinderpsychiatr, 53, 3-18.

[29] Stein, M.A., Mendelsohn, J., Obermeyer, W.H., Amromin, J. and Benca. R. (2001) Sleep and behavior problems in school-aged children. Pediatrics, 107, E60. doi:10.1542/peds.107.4.e60

[30] Liu, X. and Zhou, H. (2002) Sleep duration, insomnia and behavioral problems among Chinese adolescents. Psychiatry Research, 111, 75-85. doi:10.1016/S0165-1781(02)00131-2

[31] Johnson, E.O., Chilcoat, H.D. and Breslau, N. (2000) Trouble sleeping and anxiety/depression in childhood. Psychiatry Research, 94, 93-102. doi:10.1016/S0165-1781(00)00145-1

[32] Gregory, A.M. and O'Connor, T.G. (2002) Sleep problems in childhood: A longitudinal study of developmental change and association with behavioral problems. Psychoneuroendocrinology, 27, 489-503.

[33] Frölich, J., Lehmkuhl, G. and Wiater, A. (2005) Sleep disorders in children with attention-deficit hyperactivity disorder-Results of a polysomnographic study. Z Kinder Jugendpsychiatr Psychother, 33, 205-216. doi:10.1024/1422-4917.33.3.205

[34] Hoedlmoser, K., Kloesch, G., Wiater, A. and Schabus, M. (2010) Self-reported sleep patterns, sleep problems, and behavioral problems among school children aged 8 - 11 years. Somnologie-Schlafforschung und Schlafmedizin, 14, 23-31.

[35] Smedje, H., Broman, J.E. and Hetta, J. (1999) Parents' reports of disturbed sleep in 5 - 7-year-old Swedish children. Acta Paediatrica, 88, 858-865. doi:10.1080/08035259950168793

[36] Chervin, R.D., Archbold, K.H., Dillon, J.E., Pituch, K.J., Panahi, P., Dahl, R.E. and Guilleminault, C. (2002) Association between symptoms an inattention, hyperactivity, restless legs and periodic leg movements. Sleep, 25, 213-218.
[37] Gottlieb, D.J., Vezina, R.M., Chase, C., Lesko, S.M., Heeren, T.C., Weese-Mayer, D.E., Auerbach, S.H. and Corwin, M.J. (2003) Symptoms of sleep-disordered breathing in 5-year-old children are associated with sleepiness and problem behaviors. Pediatrics, 112, 870877. doi: $10.1542 /$ peds. 112.4 .870

[38] O’Brien, L.M., Ivanenko, A., Crabtree, V.M., Holbrook, C.R., Bruner, J.L., Klaus, C.J. and Gozal, D. (2003) The effect of stimulants on sleep characteristics in children with attention deficit/hyperactivity disorder. Sleep Medicine, 4, 309-316. doi:10.1016/S1389-9457(03)00071-6

[39] Mulvaney, S.A., Goodwin, J.L., Morgan, W.J., Rosen, G.R., Quan, S.F. and Kaemingk, K.L. (2005) Behavior problems associated with sleep disordered breathing in school-aged children - The Tucson children's assessment of sleep apnea study. Journal of Pediatric Psychology, 31, 322-330. doi:10.1093/ipepsy/jsj035

[40] Urschitz, M.S., Eitner, S., Wolff, J., Guenther, A., Urschitz-Duprat, P.M., Schlaud, M. and Poets, C.F. (2007) Risk factors for sleep-related hypoxia in primary school children. Pediatric Pulmonology, 42, 805-812. doi:10.1002/ppul.20658

[41] Ersu, R., Arman, A.R., Save, D., Karadag, B., Karakoc, F., Berkem, M. and Dagli. E. (2004) Prevalence of snoring and symptoms of sleep-disordered breathing in primary school children in Istanbul. Chest, 126, 19-24. doi:10.1378/chest.126.1.19

[42] Frölich, J. And Lehmkuhl, G. (1998) Diagnostik und Differentialdiagnostik von Schlafstörungen im Kindesalter. Fortschr Neurol Psychiatr, 66, 553-569.

[43] Laberge, L., Carrier, J., Lespérance, P., Lambert, C., Vitaro, F., Tremblay, R.E. and Montplaisi, J. (2000) Sleep and circadian phase characteristics of adolescent and young adult males in a naturalistic summertime condition. Chronobiology International, 17, 489-501. doi:10.1081/CBI-100101059 